\title{
Quantitative imaging of hybrid chiral spin textures in magnetic multilayer systems by Lorentz microscopy
}

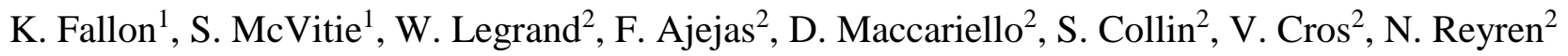 \\ ${ }^{1}$ Scottish Universities Physics Alliance, School of Physics and Astronomy, University of Glasgow, Glasgow, \\ G12 8QQ, United Kingdom
}

${ }^{2}$ Unité Mixte de Physique, CNRS, Thales, Univ. Paris-Sud, Université Paris-Saclay, 91767, Palaiseau, France

Chiral magnetic textures in ultrathin perpendicularly magnetised multilayer film stacks with an interfacial Dzyaloshinskii-Moriya interaction have been the focus of much research recently. The chirality associated with the broken inversion symmetry at the interface between an ultrathin ferromagnetic layer and a heavy metal with large spin-orbit coupling supports homochiral Néel domain walls and hedgehog (Néel) skyrmions. Under spin-orbit torques these Néel type magnetic structures are predicted, and have been measured, to move at high velocities. However recent studies have indicated that some multilayered systems may possess a more complex hybrid domain wall configuration, due to the competition between interfacial DMI and interlayer dipolar fields. These twisted textures are expected to have thickness dependent Néel and Bloch contributions to the domain or skyrmion walls. In this work, we use the methods of Lorentz microscopy to determine quantitatively for the first time experimentally both: i) the contributions of the Néel and Bloch components and ii) their spatial spin variation at high resolution. These are compared with modelled and simulated structures which are in excellent agreement with our experimental results. Our quantitative analysis provides powerful direct evidence of the Bloch wall component which exists in these hybrid walls and will be significant when exploiting chirality in spintronic applications.

\section{DOI: $\operatorname{xxxxxxxxxx}$}

\section{INTRODUCTION}

The Dzyaloshinskii-Moriya interaction (DMI) has proved to be of great interest in the study of magnetic materials whereby an antisymmetric exchange interaction causes rotation of neighbouring spins [1,2]. In magnetic thin film systems, this effect is prominent due to the strong spin-orbit coupling arising, in most cases, from the interfacial exchange interaction between the magnetic moments and neighbouring heavy metal atoms in a multi-layer system $[3,4]$. The rotation of spins is a result of the exchange Hamiltonian due to the DMI, given by $H=D_{i j}\left(S_{i} \times S_{j}\right)$ where $D_{i j}$ is the DMI vector and $S_{i}$ and $S_{j}$ are neighbouring spin vectors [5]. The interfacial DMI influences the chiral texture of domain walls in such systems so that a chiral Néel wall configuration is energetically favoured over the usual achiral divergence free Bloch rotation of the magnetisation expected in thin films [6,7]. The handedness of this Néel twisting of spins also depends on the nonmagnetic material (which determines the direction of $\boldsymbol{D})$ as well as its position with respect to the magnetic layer, i.e. above or below [8,9]. In thin
PACS numbers: 75.50.Ak, 75.60.Jk

film systems layer combinations such as $\mathrm{Pt} / \mathrm{Co} / \mathrm{Ir}$ have been used to demonstrate chiral magnetic textures with a $|\boldsymbol{D}|$ value up to $2 \mathrm{~mJ} / \mathrm{m}^{2}$ [10]. By creating multilayer repeats of these structures, for specific conditions, homochiral walls can be obtained and skyrmions can be stabilised in all layers - due to the combination of DMI and the influence of dipolar interaction in such PMA systems. Indeed, these magnetic/metallic layer systems are likely to have potential applications in future spintronic based data storage and logic devices which would exploit the efficient manipulation of Néel type domain walls and skyrmions [11-16]. However, recent studies have found the existence of hybrid domain walls in multilayer systems in which the interlayer interactions overcome the DMI [17-19]. This type of wall has a three-dimensional structure where, in some cases, internal magnetic layer(s) have a Bloch wall configuration which is situated between Néel wall surface layer(s) with opposite chirality and results in a flux closure configuration through the thickness of the multilayer. This hybrid structure has been inferred by simulations and imaging of the 
sense of rotation (or effective chirality) in the top layers through circular dichroism (CD) in x-ray resonant magnetic scattering (XRMS) [17]. This study also demonstrated, through simulation, the profound effect that this hybrid structure may have on the motion of walls and/or skyrmions under spin polarised currents; this is a conclusion also reached by a recent theoretical study which also outlines an analytical model for the hybrid structure [18]. In a separate study, simulations and nitrogen vacancy centre spin reconstruction were used to probe the wall structure of a multilayer from measurement of its stray field [19]. Our aim in this paper is to directly image the Bloch structure of the hybrid wall for the first time using Lorentz TEM and to quantify the contributions for each wall type.

A number of microscopy methods have been used to determine the magnetic texture, domain wall type and to characterise length scales of both domain walls and skyrmions in such multilayer systems. These include magnetic force microscopy (MFM) [20,21] spin polarised scanning tunnelling microscopy (SP-STM) [22], scanning transmission X-ray microscopy (STXM) $[10,23]$, spin polarised low energy electron microscopy (SPLEEM) [24], magnetic transmission soft X-ray microscopy (MTXM) [23] and X-ray photoemission electron microscopy (XPEEM) [25]. The technique of Lorentz TEM is used in this study and has previously been utilised in a number of investigations into the structure and magnetic behaviour of domain walls and skyrmions in chiral systems [26-28]. Specifically, it has been used to identify whether walls in multilayer materials with chiral texture are of Néel type [29] and also to spatially resolve domain wall widths which may only be ten nanometres or less [31]. CD-XRMS, X-PEEM, SPLEEM and (SP)STM are all surface-sensitive techniques revealing magnetization texture in the top layer(s) only. Lorentz TEM is advantageous as it is sensitive to all layers, providing an averaged projection of the magnetic textures through the thickness. Combining information from these different techniques allows one to build a threedimensional model of the magnetization texture. In this paper we detail quantitative measurements of the integrated induction using the methods of Lorentz TEM. These results, which with careful image interpretation and comparison to models, provide confirmation that the walls in the multi- repeat system studied here are consistent with truly hybrid walls possessing both Bloch and Néel components.

\section{RESULTS}

Three multilayer samples were prepared by dc magnetron sputtering. The layer structure studied

is

$\mathrm{Ta}(10) / \mathrm{Pt}(8)[\mathrm{Co}(T) / \mathrm{Ru}(1.4) / \mathrm{Pt}(0.6)] \times N / \mathrm{Pt}(2.4)$,

where the numbers are the layer thicknesses in $\mathrm{nm}$ and the substrate is on the left hand side. The parameters $T$ and $N$ are the magnetic thickness in $\mathrm{nm}$ and the number of repeat layers respectively (with combinations $(T ; N)=(1.2 ; 5),(1.4 ; 10)$ and $(1.6 ; 15))$. In the following, for convenience, we refer to the three samples by the number of magnetic layers present only, i.e. $5 \times, 10 \times$ and $15 \times$. Note however that reported properties do depend on the details of the structure, and not only $N$. Alternating gradient field magnetometry (AGFM) suggests that all three samples support out-of-plane domains; for the $5 \times$ and $10 \times$ samples, the origin is PMA from the interface but in the $15 \times$ sample, because of the Co thickness, the magnetic anisotropy favours in-plane magnetization but outof-plane domains are stabilised by dipolar interactions. The $1.4 \mathrm{~nm} \mathrm{Ru}$ layer is used in conjunction with varying Co layer thickness to ensure ferromagnetic (RKKY) coupling between the individual Co layers in each multilayer [32]. As RKKY coupling is an interfacial effect, the samples with thinner Co layers experience the strongest interlayer coupling: the $15 \times$ sample (with $1.6 \mathrm{~nm}$ Co layers) has a weaker coupling than the $5 x$ sample (with $1.2 \mathrm{~nm}$ Co layers). However, in any case, the interlayer exchange coupling is small compared to the intra-layer direct exchange $A$. For the TEM studies the samples were deposited on thin $\mathrm{Si}_{3} \mathrm{~N}_{4}$ membranes which formed electron transparent $100 \mu \mathrm{m}$ square windows on a thicker opaque silicon support. From previous work $[17,33]$ and as will be detailed later, it is expected that the $5 \times$ is likely to support only Néel type walls whereas the $10 \times$ and $15 \times$ samples are expected to have hybrid domain walls, i.e. with both Néel and Bloch contributions.

Before presenting the experimental Lorentz TEM images, we first set out the expected difference between imaging pure Néel and hybrid Néel-Bloch domain walls. We also detail the reasons that the quantitative analysis confirms the 
contribution from the Bloch and Néel components in each case. (a)
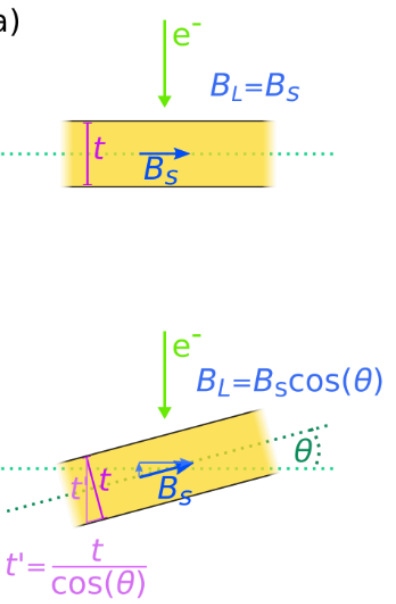

(b)
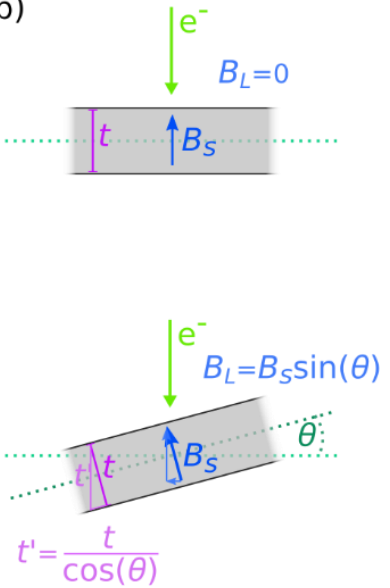

Figure 1. (a-b) Schematic figures to show to orientation of the electron beam in the TEM for films with (a) planar and (b) perpendicular magnetisation. In both cases the films have saturation induction, $B_{s}$, and thickness, $t$, and are shown untilted and tilted by an angle $\theta$. The projected thickness is indicated as $t$ '. Lorentz microscopy is sensitive to the magnetic induction perpendicular to the trajectory of the electron beam, this quantity is labelled as $B_{L}$.

Firstly, we discuss the Fresnel mode of Lorentz TEM whereby the imaging lens is defocused to reveal domain walls as black and white lines, visible due to the Lorentz deflection of the electron beam. For materials with out-of-plane domains this often requires tilting the sample from its normal position (where the film plane is perpendicular to the electron beam). Tilting results in a deflection of the beam due to the Lorentz force in the out-of-plane domains, and therefore provides contrast. This has been considered previously but only when walls have been of pure Néel or Bloch type [29]. In the case of hybrid walls, we need to consider how both components contribute to the Lorentz deflection. This is done with the aid of Figs. 1(a) and (b) which illustrate the effect of tilting on the two components of induction. Assuming magnetic films with constant saturation induction in each case, the important consideration in Lorentz TEM is the induction component perpendicular to the electron beam ( $B_{L}$ in the figures) integrated along its path. Therefore, for a sample with in-plane magnetisation of thickness $t$ and saturation induction $B_{s}$, we see that at normal incidence this integrated induction will be $B_{s} t$ - as shown in upper Fig. 1(a). However, when the sample is tilted by an angle $\theta$, as illustrated in lower Fig. 1(a), the orthogonal induction component becomes $B_{s} \cos \theta$. In this case, the projected thickness presented to the beam is $t / \cos \theta$ resulting in a total integrated induction which is also $B_{s} t$. By contrast, an untilted film with out-of-plane domains gives an integrated induction component which is zero (Fig. 1(b) upper) as the induction vector is parallel to the electron beam. When tilted (Fig. 1(b) lower) the component of induction and the projected thickness become $B_{s} \sin \theta$ and $t / \cos \theta$ respectively, meaning the integrated induction is then $B_{s} t \tan \theta$. With this information we now discuss what to expect from calculated Fresnel images.

In order to do this, we present a model of the magnetisation of a closely spaced pair of domain walls (three domains) which is shown in Fig. 2(ac), we chose this configuration as it matches the best conditions for the experimental imaging of worm domains under external out-of-plane magnetic field. The walls themselves are separated by $60 \mathrm{~nm}$ and each wall was created with a 1D $\tanh (x / \Delta)$ function with a width parameter of $\Delta=$ $15 \mathrm{~nm}$, a typical value. The model for pure Néel (Bloch) type walls is shown in the top (bottom) third of Fig. 2(a-c). The model for a hybrid wall with $10 \%$ Bloch to $90 \%$ Néel (representing for example a ten-repeat multilayer with nine layers Néel type and one layer Bloch type) is shown in the lower half of Fig. 2(a-c) and has $\left|M_{x}\right| \leq 0.9 M_{s}$ and $\left|M_{y}\right| \leq 0.1 M_{s}$. These are simple one-layer, thicknessaveraged models as in Lorentz TEM we measure a projection of magnetic induction through the thickness. Thus, it must be noted that a Fresnel image of a hybrid domain wall (varying $\mathrm{M}_{\mathrm{x}}$ and $\mathrm{M}_{\mathrm{y}}$ through the thickness) is identical to an intermediate domain wall (constant but non-zero $\mathrm{M}_{\mathrm{x}}$ and $\mathrm{M}_{\mathrm{y}}$ through the thickness) - sketches of these two configurations are provided in supplemental material S1 [30]. The calculated Fresnel images for Néel, hybrid and Bloch domain walls are shown in Fig. 2(d-f) for three sample tilts: $+20^{\circ}, 0^{\circ}$ and $-20^{\circ}$. Here the tilt axis is perpendicular to the length of the walls and in the plane of the film as indicated. 


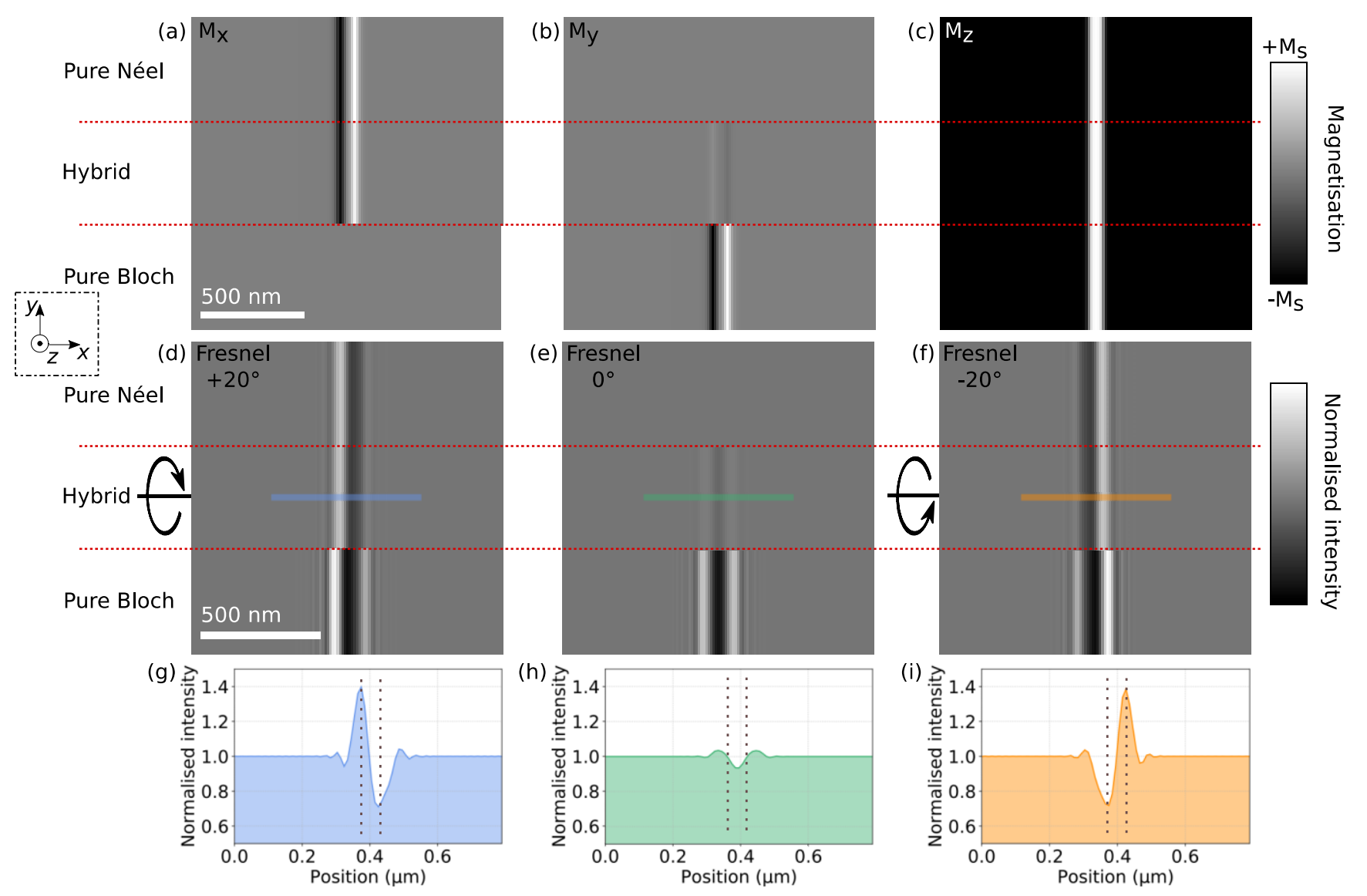

Figure 2. (a-c) $M_{x} M_{y}$ and $M_{z}$ components, constructed from a simple 1D hyperbolic tangent model, of two closely spaced domain walls. The upper third, above the top red dividing line, (a-c) models pure Néel walls whilst the middle third models hybrid Bloch/Néel walls, with a Bloch to Néel ratio of 0.1 to 0.9 , and the lower third models pure Bloch type walls. Calculated Fresnel images (d-f) of the pure Néel (upper), hybrid (middle) and Bloch (lower) wall taken at tilt angles of $+20^{\circ}, 0^{\circ}$ and $-20^{\circ}$ respectively, about axis indicated by arrowhead. Intensity line traces from the middle part (i.e. the hybrid wall) of images (d-f) are shown in (g-i). The dashed lines on the line traces correspond to the centre positions of the domain walls.

It has already been proven that the $M_{x}$ component of a pure Néel wall gives no image contrast because the wall magnetisation is divergent [29]. The magnetisation of such a wall has an associated demagnetizing field which results in net zero integrated induction - thus its only contribution is an effective reduction of $B_{s}$. Therefore, as shown in the upper part of Fig. 2(df), only contrast arising from the $\mathrm{M}_{\mathrm{z}}$ component is observed when the sample is tilted. At zero tilt, Fig. 2(e), no contrast is observed from the pure Néel walls. Additionally, contrast is seen to reverse when tilted in opposite directions, compare upper Fig. 2(d) and (f). In the case of the hybrid walls, the calculated contrast is shown in the middle part of Fig. 2(d-f). With simple visual inspection the images of tilted samples with pure Néel and hybrid walls, Fig. 2(d,f), are difficult to distinguish. However, line profiles indicate that peaks of left and right domains have different magnitude due to the Bloch contribution. There is an observable difference between the untilted images, Fig. 2(e), with contrast visible in the lower part of Fig. 2(e) from the hybrid walls only, although this contrast is notably weaker than in the tilted images. These differences and similarities between Fresnel images of pure and hybrid walls are explained by considering how the integrated magnetic induction of each component behaves with tilt - as was shown and discussed regarding Fig. 1. In the case of the Néel wall, the out-of-plane component at a tilt of $20^{\circ}$ will give an effective integrated induction of $0.36 B_{s} t$. Whereas in the case of the hybrid wall at zero tilt, the contrast arises from the Bloch component $\left(0.1 \mathrm{M}_{\mathrm{S}}\right)$ which translates to an integrated induction of $0.1 B_{s} t$. This contribution will not change with the tilt and so the out-of-plane component dominates the contrast at the tilts shown, explaining why the tilted images of pure Néel walls and hybrid walls appear almost indistinguishable by eye. The signature contrast in the untilted hybrid case arises entirely from the Bloch component of the wall. The magnitude of the contrast is important in helping to identify that it is 
a hybrid wall and not a pure Bloch wall. This is illustrated by the lower third of Fig. 2(e) - which shows a calculated Fresnel image of an untilted pure Bloch wall. In this part of the image, the contrast is ten times higher than the central portion of Fig. 2(e) (i.e. the contrast is due to $B_{s} t$ rather than $\left.0.1 B_{s} t\right)$. Additionally, for the pure Bloch case Fresnel images calculated with a tilt of $\pm 20^{\circ}$ (lower Fig. (d) and (f)) are not dominated by domain contrast; the Bloch wall signal leads to significant image asymmetry even at the high tilt angle of $20^{\circ}$.

Experimental images were obtained in Fresnel mode using a JEOL ARMcF operated at $200 \mathrm{kV}$ [34]. Additionally, we used a pixelated detector, Medipix3 with a Quantum Detectors Merlin read out system, to acquire images rather than a traditional CCD camera due to superior noise performance [35]. In Fig. 3, we show a low magnification image from the $15 \times$ sample with an applied out-of-plane field of $270 \mathrm{mT}$ in an untilted orientation with respect to the opaque silicon frame that forms part of the substrate; part of the frame is visible in this image as the large black areas. Applying this field results in long isolated worm domains corresponding to wall pairs which are good for contrast analysis, much more convenient than the demagnetised state for which domain walls are present densely all over the sample.

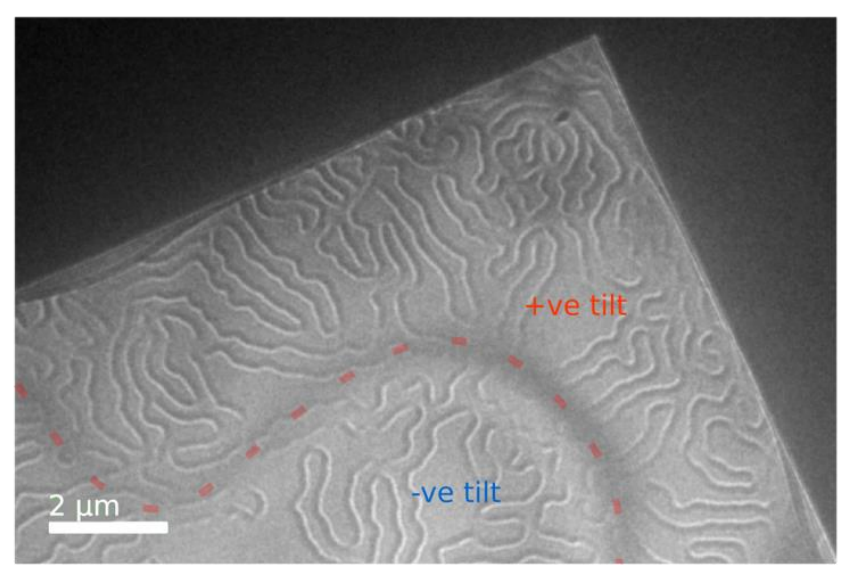

Figure 3. Low magnification Fresnel image of $15 \times$ layer sample with closely spaced pairs of domain walls. The sample is in the untilted position however the film on the transparent membrane is buckled and therefore tilted with respect to the beam. The dashed red line marks a bend contour and shows where the effective tilt with respect to the electron beam changes from negative to positive and the contrast is seen to reverse.

Interestingly, although the sample is nominally untilted, we notice that magnetic contrast is clearly visible throughout the membrane. It looks to be from narrow reverse domains which have been nucleated in a worm-like geometry. This indeed is very similar to the wall model shown in Fig. 2. However, it is important to note that distinct black/white contrast that can be seen in Fig. 3 is reversed in two regions separated by a diffuse dark line which is indicated by a dashed red line in the image. This s-shaped boundary is a bend contour and its presence indicates that the flexible membrane shows buckling in this region, which effectively results in a local tilting of the film with respect to the electron beam with the red line separating areas of opposite local tilt. This conclusion is supported by atomic force microscope (AFM) data given in supplemental material S2 which confirms membrane surface tilts of approximately $\pm 15^{\circ}$ relative to the silicon frame. Thus, the two areas either side of the red line in Fig. 3(a) show contrast of the same nature as observed in the tilted images of the model in Figs. 2(d) and (f). This shows that the contrast here is dominated by the out-of-plane domains, as expected for either Néel or hybrid walls with a small Bloch contribution.

By using a tilt-rotate specimen rod in the TEM we can orientate the sample in a direction that allows us to vary the tilt perpendicular to the wall length and observe how the contrast changes. The absolute value of the tilt of the multi-layered structure with respect to the beam thus corresponds to the local tilt due to buckling, added to the microscope rod tilt. By slowly varying the tilt, we were able get a reversal of the contrast from the outof-plane domains and then image the area at the cross-over point, which was taken as the effective untilted image. The Fresnel images at effective positive, zero and negative tilt are shown in Figs. $4(a-c)$. Note that the "zero" tilt image was at a tilt of $-9.8^{\circ}$ with respect to the notional flat plane of the membrane (i.e. the silicon opaque surface). Only the area indicated by the red oval is at zero tilt with the rest of the field of view at various levels of positive tilt with respect to the beam, this is again due to the buckling of the membrane. In Fig. 4, a clear reversal of the contrast is visible, white/black in Fig. 4(a) and black/white in Fig. 4(c). In Fig. 4(b) the contrast in the area indicated by the red oval is indeed going through a change along the wall pair. Moreover, the intensity here is considerably less 


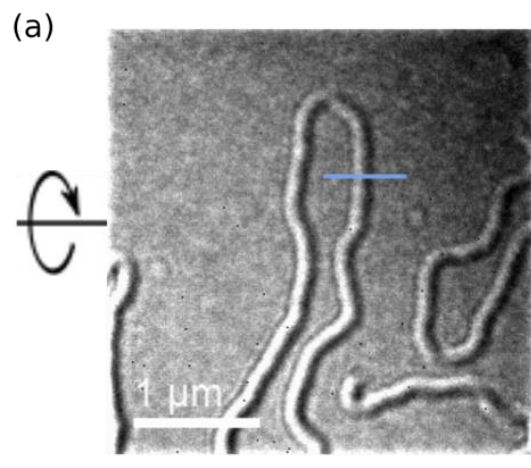

(d)

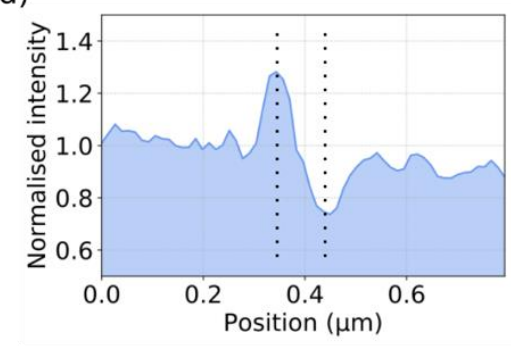

(b)

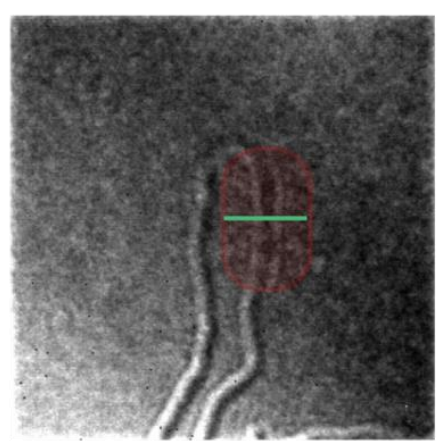

(e)

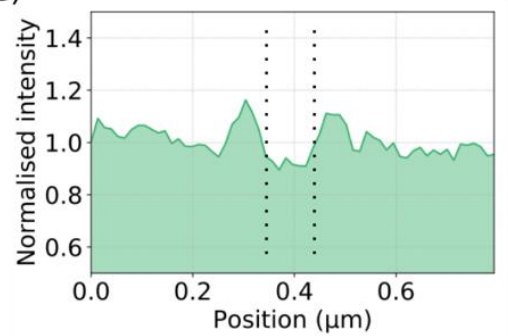

(c)

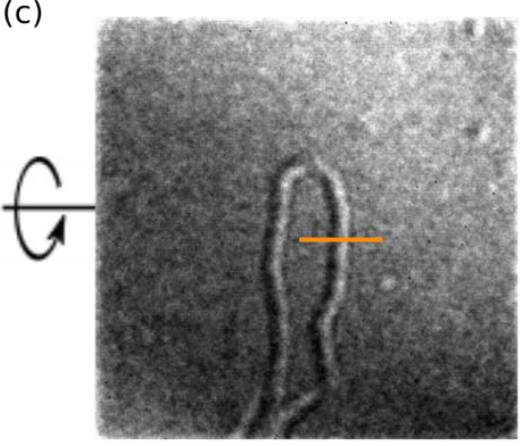

(f)

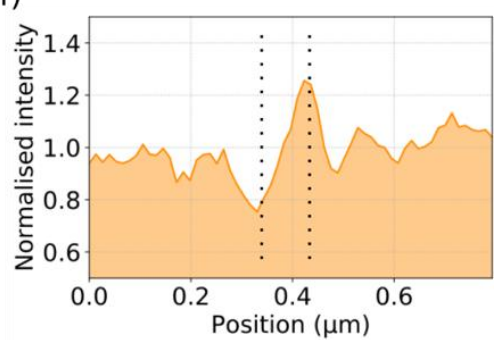

Figure 4. Fresnel images of $15 \times$ layer sample with closely spaced pairs of domain walls with varying local tilt at the coloured lines to be (a) $+5^{\circ}$, (b) $0^{\circ}$ and (c) $-5^{\circ}$. The corresponding intensity line traces from these images, averaged over 10 lines, are shown in (d-f). The dashed lines on the line trace are to guide the eye and indicate the central position of the domain wall with respect to the Fresnel image contrast. In Fresnel images the important feature is the contrast level relative to the background, therefore the line traces have been normalised to the background value.

To illustrate the variation, intensity line traces taken from these three images are shown in Figs. 4(d-f) with the contrast normalised to the background intensity to allow for direct comparison of the wall contrast in the three images. These line traces are in excellent agreement with the model suggested for the hybrid wall structure in Fig. 2. In themselves these Fresnel images are not quantitative and do not unambiguously prove that the walls have a hybrid structure. However, they do indicate that there is Bloch character to the walls and they are not of purely Néel type. The reduced intensity certainly suggests that the walls may be hybrid, but we rely on quantitative Lorentz imaging and micromagnetic simulations, described in the next section, to prove this. Fresnel images were also taken of the $10 \times$ and $5 \times$ samples; these are shown and discussed in supplemental material S3\&4 [30]. In summary, the images of the $10 x$ sample appear similar to the $15 \times$, showing a definite degree of Bloch character, where the images from the $5 \times$ sample appear consistent with a pure Néel wall structure. were imaged using the technique of differential phase contrast (DPC) in scanning TEM (STEM), this also utilised the Medipix3 detector [36]. Using this detector as opposed to a standard quadrant detector allows more precise measurement of the shifts of the unscattered central diffraction disk due to the Lorentz deflection of the electron beam. In the case of multilayer films with out-of-plane domains, experience shows that the pixelated detector is necessary to get good magnetic contrast, especially for polycrystalline films with small Lorentz deflection angles [31]. The difficulties related to imaging the multilayer samples specific to this study are discussed in supplemental material S5 [30]. The beam shifts measured by DPC are converted into quantitative integrated induction maps which allow quantification of the contributions from the out-of-plane domains and Bloch wall components.

In order to use DPC to determine quantitatively the Bloch contribution to the hybrid domain walls, two image sets were acquired. One at local zero tilt and the other of the same area but obtained at a non-zero relative tilt to the first image. 
For hybrid walls there are two possible contributions to the Lorentz deflection and hence integrated induction as detailed in Fig. 1. Firstly, there is the contribution from the Bloch walls, $B_{s} t_{B}$, where $t_{B}$ is the film thickness in the system associated with the Bloch wall structure. Secondly, there is the tilt dependent contribution from the outof-plane domains, $B_{s} t \tan \theta$, where $t$ is the total magnetic thickness. Assuming the tilt perfectly corresponds to normal electron incidence, the first image contains only a contribution from the Bloch walls. The second image is taken at a tilt angle $\theta$ relative to the first image, where $\theta$ is chosen to be sufficiently large so that the domain contrast clearly dominates. This second image is then used as a reference from which $B_{s} t$ is extracted. (a)

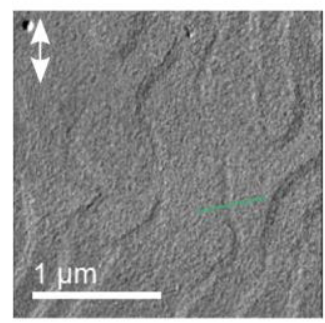

(c)

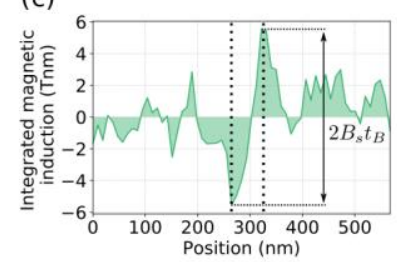

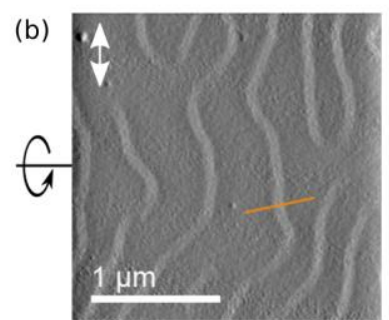

(d)

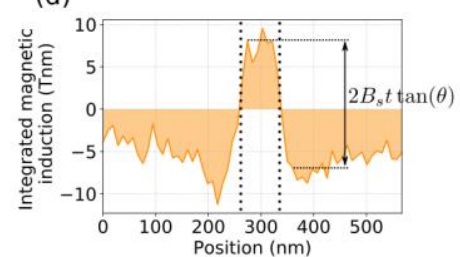

Figure 5. DPC images of the $15 \times$ layer sample taken at two different values of sample tilt. The local tilt at the coloured lines are (a) $0^{\circ}$ and (b) $+13 \cdot 2^{\circ}$. The component of induction mapped is indicated by the double headed arrow. The corresponding line traces, averaged over 20 lines, of the integrated induction from these images are shown in (c) and (d). To aid interpretation, the line traces include vertical dotted lines which indicate the position of the domain walls and horizontal dotted lines which show the salient (labelled) quantities extracted from the graphs.

DPC images from the $15 \times$ sample are shown in Fig. 5 at two different tilts and map the component of integrated induction in the direction indicated by the double-headed arrow inset on Figs. $5(a-b)$ which is close to the orientation of the wall length. Again, the zero tilt was found in the Fresnel mode prior to DPC imaging and the region at the green line in Fig. 5(a) was determined as untilted (the tilt with respect to the flat silicon substrate was
12. $2^{\circ}$ ). The image in Fig. 5(b) shows the sample tilted a further $13.2^{\circ}$ with respect to Fig. 5(a). Line traces showing the integrated induction from the same regions are given in Figs. 5(c) and (d). In Fig. 5(a), and the associated line trace in Fig. 5(c), the contrast shows a black/white contrast at the walls with the intensity change corresponding to an integrated induction $\pm B_{s} t_{B}$, as labelled on the graph. For the tilted image shown in Fig. 5(b) and the associated line trace in Fig. 5(d) the integrated induction corresponds to $\pm B_{s} t \tan \theta$ (as labelled). As $B_{s}$ is the same throughout the film and knowing the value of the tilt, $\theta$, the integrated induction ratio from these two measurements provides the ratio $t_{B} / t$ to be $0.18 \pm 0.02$. Converting this into magnetic layers suggests an equivalent of 2.7 of the 15 layers constitute the Bloch portion of the hybrid wall. The measurements from the $10 \times$ sample give a very similar ratio of $0.16 \pm 0.02$ and equates to 1.6 of the 10 layers having Bloch nature. As was previously mentioned, the methods of Lorentz microscopy do not distinguish between an intermediate Néel/Bloch wall (one with non-zero but constant $\mathrm{M}_{\mathrm{x}}$ and $\mathrm{M}_{\mathrm{y}}$ throughout the thickness) and a hybrid Néel/Bloch wall (one with varying $\mathrm{M}_{\mathrm{x}}$ and $\mathrm{M}_{\mathrm{y}}$ through the thickness) as the thickness projected magnetic induction from each is identical. However, taken in conjunction with the surface sensitive measurements by $\mathrm{x}$-ray magnetic scattering experiments from similar samples in [17] - which measured opposite handedness of Néel walls on, effectively, the top and bottom surfaces of the samples - this allow us to be certain we are imaging hybrid domain walls. As with the Fresnel images we were unable to obtain any measureable Bloch signal from the $5 \times$ sample at zero tilt, therefore this suggests a Bloch contribution below our measurement capability or is consistent with the wall being purely Néel in character.

For comparison, we performed micromagnetic simulations with parameters corresponding to these three samples - details are provided in Appendix B. Projecting the magnetisation through the thickness, the ratio $t_{B} / t$ was found to be $0.04,0.16$ and 0.19 in the simulations of the $5 \times, 10 \times$ and $15 \times$ samples respectively. This is in excellent agreement with the experimentally measured values of 0.16 and 0.19 found for the $10 \times$ and $15 \times$ samples and is also consistent with the lack of evidence of hybrid walls in the $5 \times$ sample. The small portion of Bloch 
character predicted for the $5 \times$ sample corresponds to a very slight twist away from a pure Néel configuration in the top most layer of the simulation and is not an indication of a hybrid type wall. This twist appears in simulations where the DMI energy is only just stronger than the dipolar effects and is likely below our measurement capability. Cross-sectional vector plots of the thickness varying magnetisation, calculated by micromagnetic simulations, are presented in supplemental material S6 [30]. Due to the excellent correlation of the simulations (performed with accurate material parameters for all samples) with the experimental TEM measurements, we believe these vector plots to be representative of the complex hybrid type domain walls supported samples 1 and 2 .
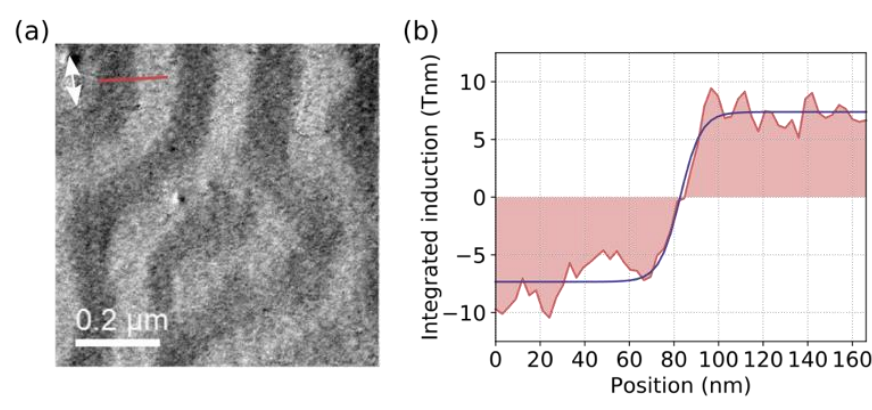

Figure 6. (a) DPC image taken of the $15 \times$ sample tilted and in a demagnetized state close to remanence, with component of induction mapped indicated by the double headed arrow. The sample is tilted to provide strong contrast from the out-of-plane domains. (b) Integrated induction line trace taken from red line in (a) showing domain wall profile and its fit to a hyperbolic tangent function, see text for details.

The spatial resolution of DPC allows the profile of the narrow domain walls to be imaged and the wall width to be measured. In Fig. 6(a), we show a DPC image of the $15 \times$ sample in a demagnetised state close to remanence together with an integrated induction profile in Fig. 6(b). After deconvolution with a Gaussian function matching the imaging probe, the line trace can be fitted to a standard hyperbolic tangent function, $B_{s} \tanh (x / \Delta)$, giving a measure of the thicknessaveraged domain wall width by the fit parameter $\Delta$. This procedure identified an average $\Delta=11 \pm 1 \mathrm{~nm}$ in the $15 \times$ sample and $\Delta=5 \pm 1 \mathrm{~nm}$ in the $10 \times$ sample. The DPC images from the $5 \times$ sample contain strong polycrystalline contrast which obscures the magnetic contrast and prevents reliable extraction of the wall width - this is due to the small magnetic thickness and the issues discussed in supplemental material S5 [30].

TABLE I. Summary of the width parameter $\Delta$, and ratio of Bloch thickness to total magnetic thickness $t_{B} / t$, measured from each sample experimentally and calculated from micromagnetic simulations of each sample - details of the simulations are provided in Appendix B.

\begin{tabular}{|c|c|c|c|c|}
\hline \multirow[b]{2}{*}{ Sample } & \multicolumn{2}{|c|}{ Experiment } & \multicolumn{2}{|c|}{ Simulation } \\
\hline & $\Delta(\mathrm{nm})$ & $t_{B} / t$ & $\Delta(\mathrm{nm})$ & $t_{B} / t$ \\
\hline $5 \times$ & - & - & 4 & 0.04 \\
\hline $10 \times$ & $5 \pm 1$ & $0.16 \pm 0.02$ & 5 & 0.16 \\
\hline $15 \times$ & $11 \pm 1$ & $0.18 \pm 0.02$ & 10 & 0.19 \\
\hline
\end{tabular}

The salient experimental results - both the wall width parameter, $\Delta$, and the Bloch thickness to total magnetic thickness ratio, $t_{B} / t$ - are summarised in Table 1, along with the same quantities measured from micromagnetic simulations. The micromagnetic simulations are in excellent agreement with the experimental measurements from both the $10 \times$ and $15 \times$ samples whilst, as mentioned previously, the wall width cannot be measured from the $5 \times$ sample but the very small $t_{B} / t$ ratio is consistent with the lack of magnetic contrast at observed at zero tilt in experimental images. The details of the micromagnetic simulations are provided in Appendix B. Experimental determination of the domain wall width is of particular interest as it allows indirect access to the exchange stiffness, $A$, of the material which is the only material parameter not experimentally measured for input to micromagnetic simulations. In supplemental material S7 [30] we explore, with micromagnetics, how $\Delta$ evolves as $A$ is varied between 3 and $20 \mathrm{pJm}^{-1}$. Fitting the experimentally measured $\Delta$ values to the curves of $\Delta$ and $A$ suggests, for both the $10 \times$ and $15 \times$ samples, $A$ in this material is close to $12 \mathrm{pJm}^{-1}$.

\section{DISCUSSION}

Here we present a Lorentz microscopy study which allowed quantitative determination of the nature of hybrid domain walls in ultrathin multilayer stacks. These measurements are complementary to recent experimental studies which utilised surface sensitive imaging techniques and measured the Néel components [17,19]; in this paper measurement of the thickness projected magnetic induction gives sensitivity to all magnetic 
layers and allows us to confirm directly for the first time a Bloch component to these walls. We conclude that the imaged walls are truly hybrid type with a central Bloch core, and not intermediate, by comparison to previous studies and micromagnetic simulations. The simulations, tuned for each sample using material parameters extracted directly from the samples (or from very similar samples), stabilise flux closure hybrid type walls with a thickness projected Bloch component extremely comparable to that measured experimentally with DPC. Our findings show that only a small fraction, about one fifth, of the layers in the system studied have a Bloch configuration when the number of layers $N=10$ and 15 . When the number of layers is small $(N=5)$, this Bloch rotation appears not to be present. Note that the critical number of repetitions to generate hybrid textures depends not only on the number of repetitions but also upon other magnetic parameters such as effective magnetization, magnitude of $\mathrm{D}$, etc. For all three multilayer systems studied, our experimental results are in excellent agreement with micromagnetic simulations for both the ratio of Bloch to Néel wall contributions and the domain wall widths. The predicted width parameters are $10 \mathrm{~nm}$ and below, values that are easily measured by the high spatial resolution Lorentz microscopy techniques [34]. As an aside we note that the observed magnetisation configuration seen through the thickness in these multilayer films resembles the structure in walls observed previously in thick (> $60 \mathrm{~nm}$ ) single layer magnetic films almost 50 years ago but possessing planar magnetisation [37,38]. Here however the multilayer structure, with limited stiffness along the out-of-plane direction, allows twisting of the DW texture even for relatively modest thickness. Additionally, thickness dependent wall structures have also been predicted and observed in other studies of skyrmions in bulk materials. Two examples are so-called chiral bobber structures as well as Néel caps in bulk Bloch skyrmions [39-42] - it should be noted that the neither are driven by dipolar factors.

Knowledge of the detailed structure of domain walls is extremely important to predict and understand both their stability and their dynamics. We demonstrate here that the metrology provided by nanoscale quantitative imaging is necessary to fully characterise the magnetic structure of these complex domain walls. Such information is critical for predicting how spin polarized currents will interact with chiral domain walls for spintronic applications [17,18].

Data associated with this work are available from the University of Glasgow: Enlighten Data repository [44] under a CC-BY license.

\section{ACKNOWLEDGEMENTS}

The authors acknowledge financial support from European Union grant MAGicSky No. FET-Open665095 and EPSRC through grant EP/M024423/1 and from the Agence Nationale de la Recherche, France, under Grant Agreement No. ANR-17CE24-0025 (TOPSKY). Additionally, we thank Albert Fert who was initially involved in devising these film structures.

\section{APPENDIX A: EXPERIMENTS AND METHODS}

Multilayers were deposited by dc magnetron sputtering at room-temperature, under $\mathrm{Ar}$ as flow at a pressure of $0.25 \mathrm{~Pa}$. The deposition rates were calibrated prior to the present depositions by X-ray reflectivity measurements. Base pressure of the sputtering system was better than $8 \cdot 10^{-6} \mathrm{~Pa}$. The multilayers of this study have been deposited on top of $\mathrm{Ta}(10 \mathrm{~nm}) / \operatorname{Pt}(8 \mathrm{~nm})$ buffers, which allows a control over their perpendicular magnetic anisotropy, and capped with $\operatorname{Pt}(3 \mathrm{~nm})$ layers to prevent oxidation. The saturation magnetisation $\mathrm{M}_{\mathrm{s}}=1-1.2 \mathrm{MA} / \mathrm{m}$ has been obtained by averaging SQUID measurements.

All of the Lorentz microscopy images were taken using a JEOL ARM 200cF equipped with a cold field emission gun and CEOS probe aberration corrector. The Fresnel imaging was carried out in TEM mode with lens defocus of between 1 and 5 $\mathrm{mm}$. The DPC imaging was performed in STEM with a condenser (probe forming) aperture of diameter $40 \mu \mathrm{m}$. The latter gives a probe of $3.5 \mathrm{~nm}$ and a resolution of $1.75 \mathrm{~nm}$. The images providing the high-resolution domain wall profiles were acquired with a sampling pixel size of $3.0 \mathrm{~nm}$ at the highest magnification used here of $\times 250 \mathrm{k}$. The AFM data in the supplemental material was taken using a Veeco Dimension 3100 Scanning Probe Microscope operated in tapping mode with a standard non-magnetic tip. The AFM image displayed in this paper is a scan over an area of $30 \times 30 \mu \mathrm{m}$. 


\section{APPENDIX B: DETAILS OF MICROMAGNETIC SIMULATIONS}

Domain profile simulations were performed with Mumax3 [43]. The cell size was fixed as $0.25 \times 0.25 \times 0.2 \mathrm{~nm}$ along $\mathrm{x}$ (wall normal), $\mathrm{y}$ (wall length) and $\mathrm{z}$ (layer planes normal) directions, respectively. For demagnetized systems, a unique period of domains was simulated, which was extended through periodic boundary conditions in the $\mathrm{x}$ and $\mathrm{y}$ directions, extending the system to 32 domain periods and $4.096 \mu \mathrm{m}$ along $\mathrm{x}$ and $\mathrm{y}$ directions respectively. Then the magnetic configuration composed of one up domain $\left(M_{z}=M_{s}\right)$ and one down domain $\left(M_{z}=\right.$ $M_{s}$ ) separated by domain walls (using the domain periodicity measured by Lorentz TEM) was directly relaxed to reach the minimum energy state. Parameters were Heisenberg exchange $A=10 \mathrm{pJ} / \mathrm{m}$ and saturation magnetisation $M_{s}=1 \mathrm{MAm}^{-1}$ for the $5 \times$ and $10 \times$ samples and $\mathrm{M}_{\mathrm{s}}=1.2 \mathrm{MAm}^{-1}$ for the $15 \times$ sample. Simulations were trialled with different values of $M_{s}$, over the range identified experimentally by SQUID, the above values were decided on as they provided the best fit to the experimental data. The magnetic thickness of the $N$ layers and thickness of vacuum spacing are chosen to match the ones of each multilayer. Uniaxial perpendicular anisotropy $K_{u}=0.829 \mathrm{MJm}^{-3}, 0.711$ $\mathrm{MJm}^{-3}$ and $0.622 \mathrm{MJm}^{-3}$; DMI parameter $D=0.825$ $\mathrm{mJm}^{-2}, 0.707 \mathrm{mJm}^{-2}$ and $0.619 \mathrm{mJm}^{-2}$ were chosen for samples with Co layer thickness $T=1.2 \mathrm{~nm}, 1.4$ $\mathrm{nm}$ and $1.6 \mathrm{~nm}$ respectively. They thus match with the interfacial perpendicular anisotropy and interfacial DMI value measured from a similar sample with $T=1.1 \mathrm{~nm}$ considering an inverse thickness dependence.

[1] I. Dzyaloshinsky, A thermodynamic theory of "weak" ferromagnetism of antiferromagnetics, J. Phys. Chem. Solids 4, 241-255 (1958).

[2] T. Moriya, Anisotropic superexchange interaction and weak ferromagnetism, Phys. Rev. 120, 91-98 (1960).

[3] A. N. Bogdanov, and U. K. Roßer, Chiral symmetry breaking in magnetic thin films and multilayers, Phys. Rev. Lett. 87, 037203 (2001).

[4] M. Bode, M. Heide, K. von Bergmann, P. Ferriani, S. Heinze, G. Bihlmayer, A. Kubetzka, O. Pietzsch, S. Blügel, and R. Wiesendanger, Chiral magnetic order at surfaces driven by inversion asymmetry, Nature 447, 190-193 (2007).

[5] A. Fert, Magnetic and Transport Properties of Metallic Multilayers, Mater. Sci. Forum 59-60, 439-480 (1990).

[6] A. Thiaville, S. Rohart, É. Jué, V. Cros, and A. Fert. Dynamics of Dzyaloshinskii domain walls in ultrathin magnetic films, Europhys. Lett. 100, 57002 (2012).

[7] N. Nagaosa, and Y. Tokura, Topological properties and dynamics of magnetic skyrmions, Nat. Nanotechnol. 8, 899-911 (2013).

[8] G. Chen, T. Ma, A. T. N'Diaye, H. Kwon, C. Won, Y. Wu, and A. K. Schmid, Tailoring the chirality of magnetic domain walls by interface engineering, Nat. Commun. 4, 2671 (2013).

[9] A. Hrabec, N. A. Porter, A. Wells, M. J. Benitez, G. Burnell, S. McVitie, D. McGrouther, T. A. Moore, and C. H. Marrows, Measuring and tailoring the Dzyaloshinskii-Moriya interaction in perpendicularly magnetized thin films, Phys. Rev. B 90, 020402 (2014).

[10] C. Moreau-Luchaire, C. Moutafis, N. Reyren, J. Sampaio, C. A. F. Vaz, N. Van Horne, K. Bouzehouane, K. Garcia, C. Deranlot, P. Warnicke et al., Additive interfacial chiral interaction in multilayers for stabilization of small individual skyrmions at room temperature, Nat. Nanotechnol. 11, 444-448 (2016).

[11] R. Wiesendanger, Nanoscale magnetic skyrmions in metallic films and multilayers: a new twist for spintronics, Nat. Rev. Mater. 1, 16044 (2016).

[12] A. Fert, N. Reyren, and V. Cros, Magnetic skyrmions: advances in physics and potential applications, Nat. Rev. Mater. 2, 17031 (2017).

[13] I. M. Miron, T. Moore, H. Szambolics, L. D. Buda-Prejbeanu, S. Auffret, B. Rodmacq, S. Pizzini, J. Vogel, M. Bonfim, A. Schuhl et al. Fast current-induced domain-wall motion controlled by the Rashba effect, Nature Mater. 10, 419 (2011).

[14] K. S. Ryu, L. Thomas, S. H. Yang, and S. S. P. Parkin, Current induced tilting of domain walls in high velocity motion along perpendicularly magnetized micron-sized Co/Ni/Co racetracks, Appl. Phys. Express 5, 093006 (2012).

[15] P. P. J. Haazen, E. Murè, J. H. Franken, R. Lavrijsen, H. J. M. Swagten, and B. Koopmans, Domain wall depinning governed by the spin Hall effect, Nat. Mater. 12, 299 (2013). 
[16] E. Martinez, S. Emori, and G. S. D. Beach, Current-driven domain wall motion along high perpendicular anisotropy multilayers: The role of the Rashba field, the spin Hall effect, and the Dzyaloshinskii-Moriya interaction, Appl. Phys. Lett. 103, 072406 (2013).

[17] W. Legrand, J.-Y. Chauleau, D. Maccariello, N. Reyren, S. Collin, K. Bouzehouane, N. Jaouen, V. Cros, and A. Fert, Hybrid chiral domain walls and skyrmions in magnetic multilayers, Sci. Adv. 4, eaat0415 (2018).

[18] I. Lemesh, and G. S. D. Beach, Twisted domain walls and skyrmions in perpendicularly magnetized multilayers, Phys. Rev. B 98, 104402 (2018).

[19] Y. Dovzhenko, F. Casola, S. Schlotter, T. X. Zhou, F. Büttner, R. L. Walsworth, G. S. D. Beach, and A. Yacoby, Magnetostatic twists in roomtemperature skyrmions explored by nitrogenvacancy center spin texture reconstruction, Nat. Commun. 9, 2712 (2018).

[20] M. Baćani, M. A. Marioni, J. Schwenk, and H. J. Hug, How to measure the local DzyaloshinskiiMoriya interaction in skyrmion thin film multilayers. Sci. Rep 9, 3114 (2019).

[21] W. Legrand, D. Maccariello, N. Reyren, K. Garcia, C. Moutafis, C. Moreau-Luchaire, S. Collin, K. Bouzehouane, V. Cros, and A. Fert, Room-temperature current-induced generation and motion of sub-100 nm skyrmions, Nano Letters 17, 2703-2712 (2017).

[22] N. Romming, C. Hanneken, M. Menzel, J. E. Bickel, B. Wolter, K. von Bergmann, A. Kubetzka, and $\mathrm{R}$. Wiesendanger, Writing and deleting single magnetic skyrmions, Science 341, 636-639 (2013). [23] S. Woo, K. Litzius, B. Krüger, M.-Y. Im, L. Caretta, K. Richter, M. Mann, A. Krone, R. M. Reeve, M. Weigand et al., Observation of roomtemperature magnetic skyrmions and their currentdriven dynamics in ultrathin metallic ferromagnets, Nat. Mater. 15, 501-506 (2016).

[24] G. Chen, S. P. Kang, C. Ophus, A. T. N'Diaye, H. Y. Kwon, R. T. Qiu, C. Won, K. Liu, Y. Wu, and A. K. Schmid, Out-of-plane chiral domain wall spin structures in ultrathin in-plane magnets, Nat. Commun. 8, 15302 (2017).

[25] O. Boulle, J. Vogel, H. Yang, S. Pizzini, D. de Souza Chaves, A. Locatelli, T. O. Menteş, A. Sala, L. D. Buda-Prejbeanu, O. Klein et al., Roomtemperature chiral magnetic skyrmions in ultrathin magnetic nanostructures, Nat. Nanotech. 11, 449-454 (2016).
[26] S. Zhang, A. K. Petford-Long, and C. Phatak, Creation of artificial skyrmions and antiskyrmions by anisotropy engineering, Sci. Rep. 6, 31248 (2016).

[27] J. J. Chess, S. A. Montoya, T. R. Harvey, C. Ophus, S. Couture, V. Lomakin, E. E. Fullerton, and B. J. McMorran, Streamlined approach to mapping the magnetic induction of skyrmionic materials, Ultramicroscopy 177, 78-83 (2017).

[28] S. D. Pollard, J. A. Garlow, J. Yu, Z. Wang, Y. Zhu, and H. Yang, Observation of stable Néel skyrmions in cobalt/palladium multilayers with Lorentz transmission electron microscopy, Nat. Commun. 8, 14761 (2016).

[29] M. J. Benitez, A. Hrabec, A. P. Mihai, T. A. Moore, G. Burnell, D. McGrouther, C. H. Marrows, and S. McVitie, Magnetic microscopy and topological stability of homochiral Néel domain walls in a Pt/Co/AlO $\mathrm{A}_{x}$ trilayer, Nat. Commun. 6, 8957 (2015).

[30] See Supplemental Material at [URL] for description of intermediate and hybrid type domain walls, AFM images showing surface contortion, additional experimental Fresnel images, discussion of DPC imaging of skyrmionic multilayer materials, and micromagnetic simulations.

[31] S. McVitie, S. Hughes, K. Fallon, S. McFadzean, D. McGrouther, M. Krajnak, W. Legrand, D. Maccariello, S. Collin, K. Garcia et al., A transmission electron microscope study of Néel skyrmion magnetic textures in multilayer thin film systems with large interfacial chiral interaction, Sci. Rep. 8, 5703 (2018).

[32] P. J. H. Bloemen, H. W. van Kesteren, H. J. M. Swagten, and W. J. de Jonge, Oscillatory interlayer exchange coupling in Co/Ru multilayers and bilayers, Phys. Rev. B 50, 18 (1994).

[33] J.-Y. Chauleau, W. Legrand, N. Reyren, D. Maccariello, S. Collin, H. Popescu, K. Bouzehouane, V. Cros, N. Jaouen, and A. Fert, Chirality in magnetic multilayers probed by the symmetry and the amplitude of dichroism in x-ray resonant magnetic scattering, Phys. Rev. Lett. 120, 037202 (2018).

[34] S. McVitie, D. McGrouther, S. McFadzean, D. A. MacLaren, K. J. O'Shea, and M. J. Benitez, Aberration corrected Lorentz scanning transmission electron microscopy. Ultramicroscopy 152, 57-62 (2015).

[35] J. A. Mir, R. Clough, R. MacInnes, C. Gough, R. Plackett, I. Shipsey, H. Sawada, I. MacLaren, R. 
Ballabriga, D. Maneuski et al., Characterisation of the Medipix3 Detector for 60 and $80 \mathrm{keV}$ electrons, Ultramicroscopy 182, 44 (2017).

[36] M. Krajnak, D. McGrouther, D. Maneuski, V. O'Shea, and S. McVitie, Pixelated detectors and improved efficiency for magnetic imaging in STEM differential phase contrast, Ultramicroscopy $\mathbf{1 6 5}$, 42-50 (2016).

[37] A. E. LaBonte, Two-dimensional Bloch-type domain walls in ferromagnetic films, J. Appl. Phys. 40, 2450 (1969).

[38] A. Hubert, Stray-field-free and related domain wall configurations in thin magnetic films (II), Phys. Status Solidi B 38, 699-713 (1970).

[39] F. N. Rybakov, A. B. Borisov, S. Blügel, and N. S. Kiselev, New type of stable particle-like states in chiral magnets, Phys. Rev. Lett. 115, 117201 (2015).

[40] F. Zheng, F. N. Rybakov, A. B. Borisov, D. Song, S. Wang, Z.-A. Li, H. Du, N. S. Kiselev, J.
Caron, A. Kovács et al., Experimental observation of chiral magnetic bobbers in B20-

type $\mathrm{FeGe}$, Nature Nanotechnol. 13, 451455 (2018).

[41] D. McGrouther, R. J. Lamb, M. Krajnak, S. McFadzean, S. McVitie, R. L. Stamps, A. O. Leonov, A. N. Bogdanov, and Y. Togawa, Internal structure of hexagonal skyrmion lattices in cubic helimagnets, New J. Phys. 18, 095004 (2016).

[42] A. O. Leonov, Y. Togawa, T. L. Monchesky, A. N. Bogdanov, J. Kishine, Y. Kousaka, M. Miyagawa, T. Koyama, J. Akimitsu, Ts. Koyama et al., Chiral surface twists and skyrmion stability in nanolayers of cubic helimagnets, Phys. Rev. Lett. 117, 087202 (2016).

[43] A. Vansteenkiste, J. Leliaert, M. Dvornik, M. Helsen, F. Garcia-Sanchez, and B. Van Waeyenberge, The design and verification of MuMax3. AIP Adv. 4, 107133 (2014).

[44] Enlighten data repository at, http://dx.doi.org/10.5525/gla.researchdata.893 